I.

\title{
Mittheilungen aus dem Gebiete des einfachen und eitrigen Mittelohrkatarrhs.
}

\author{
Von \\ Dr. Louis Blau \\ in Berlin.
}

1. Sklerose der Paukenhöhlenschleimhaut; Injectionen von Chloralhydrat. Später acute eitrige Mittelohrentzündung mit eigenthümlichem Verlauf.

Herr P., Kaufmann, 36 Jahre alt, kam im April 1877 zum ersten Mal in meine Behandlung. Derselbe klagte über beiderseitige Schwerhörigkeit, welche schon seit circa 20 Jahren bestand, rechts stärker als auf dem linken Ohre war, sich allmählich entwickelt und mit der Zeit bedeutend zugenommen hatte. Starkes Sausen war zuweilen vorhanden. Sodann liess sich anamnestisch noch eruiren, dass der Kranke vor ungefähr 15 Jahren ein Ulcus durum acquirirt hatte, welchem mehrere Monate später ein Exanthem, aber keine weiteren specifischen Symptome nachfolgten. Die Untersuchung des Hörvermögens ergab, dass links ziemlich laute Sprache in der Nähe, rechts nur noch leise Sprache durch das Hörrohr verstanden wurde. Uhr links schwach beim Anlegen, deutlich vom Knochen aus, rechts nur vom Knochen; Stimmgabeln vom Scheitel aus links stärker. Das rechte Trommelfell erschien weisslich getribt, und zwar in seinem hinteren Segmente mehr als im vorderen, wo ausserdem zwei runde stecknadelkopfgrosse Verkalkungen vorhanden waren. Wölbung und Oberflächenglanz normal, Hammergriff und Processus brevis deutlich, in normaler Weise prominirend, Lichtreflex im vorderen unteren Quadranten strichförmig. Links war das Trommelfell flach, stark glänzend, durchscheinend und mit leichten circumscripten weisslichen Trübungen versehen. Das Manubrium und 
der kurze Fortsatz des Hammers waren deutlich sichtbar, indessen prominirten dieselben nicht, wie rechts und uberhaupt in der Norm, etwas nach aussen, sondern es machte vielmehr den Eindruck, als ob beide hinter dem Trommelfell gelegen wären und man sie durch das letztere nur hindurchschimmern sähe. Lichtkegel der Quere nach unterbrochen. Bei der Luftdouche durch den Katheter strömte die Luft ohne Rasseln ein, rechts in breiterem Strome als auf der linken Seite; darnach war ein Gefuhl subjectiver Erleichterung vorhanden, jedoch das Hörvermögen nicht gebessert. Beweglichkeit der Trommelfelle normal. Starker chronischer Rachen- und Nasenrachenkatarrh.

Die Therapie bestand vor Allem in regelmässiger Luftdouche durch den Katheter und Einspritzungen einer wässerigen Lösung von Chloralhydrat $(1,0: 30,0)$ in die Paukenhöhle. Die letzteren wurden wöchentlich zweimal gemacht, im Ganzen auf jeder Seite 12 Injectionen; ihre Schmerzhaftigkeit war sehr bedeutend, das Trommelfell unmittelbar darnach stark geröthet, indessen waren schon am nächsten Tage die Reactionserscheinungen ausnahmslos wieder verschwunden. Trotzdem erwies sich der Erfolg als ein recht guter, indem das Sausen wesentlich an Intensität abnahm, die Uhr am Schluss der Behandlung beiderseits auf $2 \mathrm{Cm}$. Entfernung gehört wurde und auch das Sprachverständniss, besonders auf dem linken $\mathrm{Ohr}$, entschieden gebessert erschien. Diese Besserung hielt auch weiterhin noch an, bis sich ungefähr ein Jahr später im Gefolge eines Schnupfens linkerseits aufs Neue vermehrte Schwerhörigkeit und Sausen, sowie ein Gefühl von Verlegtsein des Ohres und Kneifen in demselben einstellten. Bei der Untersuchung ergab sich der nämliche Befund wie das vorige Mal, desgleichen wuide therapentisch wieder regelmässiger Catheterismus und nach 14 Tagen ausserdem Injectionen von Chloralhydrat angewandt, wobei es sich zeigte, dass durch die Luftdouche allein zwar einige Besserung eintrat, dass diese aber erst dann wesentliche Fortschritte machte, als noch die irritirenden Einspritzungen hinzugefügt wurden. Das Gehör für die Sprache (links) war am Ende der Cur derart, dass sich Patient leicht bei einer mittellaut gefuhrten Unterhaltung betheiligen konnte, das Sausen trat kaum noch störend hervor, die tibrigen subjectiven Beschwerden waren vollkommen verschwunden. Rechts liess sich keine Veränderung im Hörvermögen constatiren, hier wurde, wie friher, die Sprache nur durch das Hörrohr verstanden.

In den nächsten 5 Jahren sah ich Herrn P. mehrere Male 
und konnte mich iberzengen, dass das Gehör sich linkerseits unverändert anf gleicher Höhe erbielt, ja nach Ablauf der letzten Cur hatte es während eines Aufenthaltes im Gebirge sogar noch eine Zunahme, ebenso wie das Sausen noch eine nachträgliche Verminderung erfahren. Am 5. März 1883 wurde ich aufs Neue zu dem Patienten gerufen, da derselbe plötzlich nach voraufgegangenem Schnupfen und Schlingbeschwerden heftige Schmerzen, Sausen und Klopfen im rechten Ohre bekommen hatte. Starke Druckempfindlichkeit vor dem Tragus und unter dem Obre, weniger in der Gegend des Warzenfortsatzes. Im Gehörgang sparsame blatig-seröse Flussigkeit; solche lag auch in dunner Schicht dem vorderen unteren Rand des Trommelfells an und zeigte hier deutliche Pulsationen. Trommelfell flach, sehr stark geröthet, glanzlos, mit einer fast das ganze vordere Segment einnehmenden Ekchymose, aber ohne Perforation. Hammergriff und Processus brevis unsichtbar. Die Behandlung bestand in einer localen Blutentziehung (zwei Blutegel vor dem Tragus, zwei am Warzenfortsatz), der innerlichen Verabreichung von Bittersalz und Warmwasserumschlägen, resp. Einträufelungen gegen die Schmerzen.

6. März. Schmerzen geringer, aber noch immer vorhanden. Jodanstrich in der Umgebung des Ohres.

7. März. Profuser dïnner röthlich-gelber Ausfluss. Perforation im vorderen unteren Trommelfellquadranten. In der Nacht waren wieder Schmerzen aufgetreten. Sorgfältige Reinhaltung des Ohres durch Politzer's Verfahren und Ausspritzungen mit Borsäurelösung.

10. März. Profuse Otorrhoe. Schmerzen im Ganzen gering, stellten sich aber noch immer zeitweise, besonders des Nachts ein.

11. März. Otorrhoe bedeutend geringer. Trommelfell unverändert stark geröthet.

13. März. In der Nacht wieder heftigere Schmerzen im Ohr. Otorrhoe sistirt, Perforation geschlossen. Trommelfell stark geröthet und verdickt. Hammergriff unsichtbar. Die durch den Katheter geblasene Luft drang bei wenig erhöhtem Druck ohne Rasseln in die Paukenhöhle ein. Verordnet wurde innerlich Morphium und die Application einer Eisblase auf das Ohr.

14. März. Die Schmerzen waren in der Nacht so heftig, dass der Kranke dieselbe ohne jeden Schlaf zubringen musste; auch am Tage dauerten sie in wenig ermässigter Intensität noch fort. Allgemeines Unwohlsein. Druckempfindlichkeit vor dem Tragus, dagegen war der Warzenfortsatz weder spontan, noch 
bei Druck schmerzhaft und seine Bedeckungen nicht geschwollen. Das Trommelfell erschien ohne Perforation, stark geröthet und verdickt, besonders in seinem hinteren Segment von dunkelblaurother Färbung. Bei der Luftdouche durch den Katheter lautes Blasegeräusch ohne Rasseln. In der Meinung, dass trotzdem Secret in der Paukenhöhle zurückgehalten sein könnte, und ausserdem, um die entzundliche Spannung zu vermindern, machte ich eine ausgiebige Incision in das hintere Trommelfellsegment. Die Operation war sehr sehmerzhaft und von reichlicher Blutung gefolgt, Eiter wurde aber nicht entleert, auch nicht bei der Luftdouche. Ausserdem verordnete ich Calomel, zweimal täglich 0,3 Grm., Morphium für die Nacht und eine Eisblase auf das Ohr.

15. März. Noch immer starke Schmerzen. Kein Ausfluss. Incisionswunde verklebt, Trommelfell intensiv geröthet. Behandlung unverändert; Jodanstrich in der Umgebung des Ohres.

16. März. Schmerzen ermässigt. Calomel aussetzen.

17. März. Schmerzen nahezu verschwunden, das Trommelfell begann abzublassen. Auch die Eisapplication wurde jetzt ausgesetat.

18. März. Keine Schmerzen mehr. Die Behandlung bestand von nun an nur noch in den Pinselungen mit Jodtinctur und regelmässiger Luftdouche durch den Katheter.

22. März. Keine Schmerzen. Trommelfell grau, mit einem leichten Stich ins Röthliche, trübe; der Hammergriff fing an, sich zu markiren. Mit den Jodpinselungen ebenfalls aufgehört.

31. März. Patient wurde entlassen. Weder Schmerz noch Sausen. Trommelfellbild und Hörschärfe wie vor der letzten Erkrankung.

Epikris e. Dass ein acuter Paukenhöhlenkatarrh beim Hinzutreten einer neuen Schädlichkeit, manchmal auch infolge einer unzweckmässigen Behandlung, z. B. mit Kataplasmen, in eine acute eitrige Mittelohrentzündung übergeht, ist eine häufiger zu beobachtende Erscheinung, dagegen dürfte das umgekehrte Verhalten, wie es in dem soeben mitgetheilten Falle stattfand, schon eher zu den Seltenheiten gehören. Denn hier begann die Krankheit als eine acute Otitis media suppurativa, dann aber verschwand der Ausfluss, die Perforation schloss sich, während die örtlichen Entzïndungssymptome und die Schmerzen an Intensität sebr bedeutend zunahmen, und von jetzt an verlief das Leiden unter dem Bilde eines schweren acuten Mittelohrkatarrhs. Daher war ich der Ansicht, die so plötzliche und ohne bekannte Ursache 
aufgetretene Verschlimmerung in dem Zustand des Patienten könnte vielleicht durch eine Eiterretention in den Räumen des Mittelohres veranlasst sein und machte, trotz des negativen Ergebnisses bei der Auscultation, die Paracentese des Trommelfells. Eiter wurde dabei nicht entleert, nichtsdestoweniger aber glaube ich mich berechtigt, der Operation einen günstigen Einfluss auf die weitere Gestaltung des Leidens zuzuschreiben, indem durch dieselbe einmal eine sehr erhebliche locale Blutentziehung bewirkt wurde und ausserdem eine Entspannung der hochgradig entziundeten Gewebe stattfand. Interessant ist, dass trotz der Schwere des Processes derselbe sich hauptsächlich auf die Pankenhöhle beschränkt erhalten hatte und eine wesentliche Betheiligung des Warzenfortsatzes nicht zn Stande gekommen war. In zweiter Linie möchte ich auf die günstige Wirkung anfmerksam machen, welche in diesem Fall die Injectionen von Chloralhydrat gehabt haben. Die Aenderung in der Hörsehärfe war, wenigstens was das linke $\mathrm{Ohr}$ anbetrifft, flir mich im höchsten Grade frappirend, viel mehr, als es sich durch die Beschreibung wiedergeben lässt, und zudem erwies sich der Erfolg auch als ein nachhaltiger, da bis zum heutigen Tage, also in 7 Jahren, noch keine Verschlechterung wieder eingetreten ist. Leider ist dieser Fall aber auch der einzige geblieben, in welchem ich eine so gitnstige Wirkung von dem Chloralhydrat beobachten konnte. Ich habe das Mittel noch bei einer grossen Anzahl mit Paukenhöhlensklerose behafteter Patienten angewandt, aber niemals die geringste Besserung, sei es des Hörvermögens oder in der Beschaffenheit der subjectiven Geräusche, erzielt. Allerdings kam auch niemals eine Verschlechterung, z. B. eine Zunahme des Ohrensausens vor, wie ich sie bei dem Gebrauche anderer irritirender Einspritzungen (selbst schon von. einer Solutio Kalii jodati $0,5: 30,0)$ wiederholentlich gesehen habe. Die Reaction hielt sich immer innerhalb mässiger Grenzen, nur einmal beobachtete ich es, dass nach der Einspritzung weniger Tropfen einer $31 / 3$ proc. Chlorallösung sich eine lebhaftere Entzündung einstellte. Der betreffende Patient, welcher bisher das in gleicher Stärke angewandte Mittel stets gut vertragen hatte, klagte nach der fünften Injection über andauernde Schmerzen, Druckempfindlichkeit vor dem Tragus, Schwerhörigkeit und Sausen, und am nächsten Tage fand ich das Trommelfell weiss, glanzlos, mit aufgelockertem Epidermistiberzuge, den Hammergriff unsichtbar und im hinteren unteren Quadranten eine kleine lineäre Perfo- 
ration, welche sich als eine feine rothe Linie präsentirte, indessen schon wieder verklebt war. Im innersten Theil des Gehörgangs und auf dem Trommelfell lag sparsamer Eiter. Uebrigens hatte dieser Zwischenfall keine schädlichen Folgen, nach 6 Tagen waren alle Störungen verschwunden und das Trommelfellbild normal. Dass bei dem oben erwähnten Kranken die eclatante Besserung in der That den Chloralinjectionen und nicht etwa der daneben regelmässig angewandten Luftdouche zuzaschreiben war, ergab sich deutlich aus der Beobachtung während der zum zweiten Male eingeleiteten Cur, indem hier, so lange ich mich auf den Catheterismus allein beschränkte, Hörschärfe und Ohrensausen nur geringe Veränderungen zeigten und erst, als ausserdem noch die Einspritzungen gemacht wurden, der ïberaus gitnstige Umschwung eintrat. - Ein dritter Punkt, auf welchen ich sodann noch hinweisen will, ist das eigenthtimliche Bild, welches der Hammergriff und der Processus brevis auf dem linken Ohr des Patienten darboten. Wie schon gesagt, fehlte die gewöhnliche Prominenz dieser Theile über das Niveau des Trommelfells, es machte tiberhaupt den Eindruck, als ob dieselben nicht dem Trommelfell eingefügt wären, sondern hinter der Membran lägen und man sie nur durch die letztere hindurch sähe. Nach der Luftdouche war in dem beschriebenen Verhalten keine Aenderung zu constatiren, wie etwa bei Loslösung des Hammergriffes von dem Trommelfell, wo jener dann vortibergehend ganz oder zum Theil verschwindet. Ich habe während meiner Thätigkeit ein ähnliches Bild nur noch einmal beobachtet, bei einem 13 jährigen Mädchen und zwar hier auf beiden Seiten in gleicher Weise vorhanden.

\section{Otitis media purulenta bei Phthisikern. Behandlung mit Jodoform.}

a) Patient, ein 25 Jahre alter Kaufmann, Phthisiker im letzten Stadium, hatte, als er in meine Behandlung kam, seit acht Tagen Schwerhörigkeit, Summen und Klopfen im linken Ohr, sowie ein schmerzbaftes Gefuhl beim Schneuzen ebendaselbst bemerkt und seit dem Tage vorher Otorrhoe. Spontane Schmerzen waren nie dagewesen. Die Untersuchung ergab eine starke Röthung und Schwellung des Trommelfells und eine etwa sandkorngrosse Perforation in dessen vorderem unterem Quadranten, auch der innerste Abschnitt des Gehörgangs nahm an der Entzindung Theil, doch war der Ausfluss nur ein sehr geringer. 
Die Therapie bestand zu Anfang in Reinigung des Ohres durch Luftdouche und Ausspritzungen und in der nachfolgenden Application von Borsäurepulver. Indessen wurde das letztere entschieden nicht vertragen. Die Entzundungserscheinungen steigerten sich in auffallender Weise, die Otorrhoe nahm an Intensität zu, auch die Perforation vergrösserte sich bis zu dem Umfange eines Hirsekorns und zudem bekam der Patient jetzt zum ersten Male während des ganzen Krankheitsverlaufes wirkliche Schmerzen. Ich ging nun zu Einträufelungen einer Solutio zinci sulfurici 0,05 bis $0,1: 30,0$ ubber, wobei zwar die Schmerzen wieder verschwanden, sonst aber im Zustande des Ohres keine Veränderung eintrat. Als drittes Mittel wurde das Jodoform angewandt. Sein Einfluss erwies sich als ein günstiger, indem nach 14 tagigem Gebrauch die Otorrhoe sistirte und die Perforation durch eine dünne Narbe geschlossen war. Leider jedoch danerte nichtsdestoweniger die Entzündung im Innern der Paukenhöhle fort, wie sich daraus ergab, dass das Trommelfell nicht abschwoll, eine blassrosarothe Färbung beibehielt und an seiner Oberfläche von injicirten, radiär verlaufenden Gefässen durchzogen war, dass ferner der Hammergriff unsichtbar blieb und sich auch die Schwerhörigkeit, das Summen und das Gefuhl von Verstopfung im Ohre nicht verloren. Die Uhr wurde jetzt vor der Luftdouche auf $8 \mathrm{Cm}$., nach derselben auf $15 \mathrm{Cm}$. Entfernung gehört. In der Folge gestaltete sich nun das Bild derart, dass Zeiten, in welehen sich die Perforation wieder offnete, und solche, in denen dieselbe geschlossen und das Befinden des Kranken das oben erwähnte war, mit einander abwechselten. Stets bewährte sich das Jodoform als dasjenige Mittel, durch welches allein ein Sistiren der Eiterung bewirkt werden konnte, und zwar variirte die $\mathrm{zu}$ diesem Resultate nothwendige Zeit zwischen 3 und 14 Tagen. Die Intervalle zwischen den einzelnen Eiterungsperioden schwankten von einigen Tagen bis zu drei Monaten. Im Ganzen zog sich das Leiden in dieser Art noch $5 / 4$ Jahre lang hin, bis der Patient seiner Lungenaffection erlag.

b) Der zweite Fall, welchen ich hier mittheilen will, betraf eine Dame von 31 Jahren, die desgleichen an Tubereulosis pulmonum litt, trotz ihrer Cavernen aber und der wiederholten Anfälle von Haemoptoë noch ein blthendes Aussehen besass. Bereits seit 4 bis 5 Jahren sollten im rechten Ohr öfters "Geschwture" aufgetreten sein, die von dem Hausarzt geöffnet wurden; seit fünf Wochen bestand jetzt Otorrhoe mit mässigen 
Schmerzen im Beginn der Krankheit. Der Ausfluss war nur gering, aber mit reichlichen Epidermisfetzen gemischt, das Trommelfell zeigte sich stark geröthet und verdickt, mit einer grossen centralen Perforation, und die Schleimhaut der inneren Paukenhöhlenwand erschien grauweiss und aufgelockert. Die Borsäureeinblasungen entfalteten anch hier eine entschieden reizende Wirkung, desgleichen vermochten tägliche Einträufelungen von Solutio argenti nitrici 2,0:30,0 mit nachfolgender Neutralisation keine Besserung herbeizuführen, während dagegen das Jodoform prompte Heilung brachte. Nach dreiwöchentlicher Anwendung des Mittels konnte die Patientin entlassen werden; der Ausfluss sowohl wie die Abstossung von Epidermisschippchen hatte aufgehört, das Trommelfell war granweiss, der Hammergriff dentlich und die durch die Perforation sichtbare Paukenhöhlenschleimhaut dünn, gelb und trocken. Als sich die Patientin 2 Jahre später wegen eines linksseitigen leichten Tubenkatarrhs wieder vorstellte, war ich in der erfreulichen Lage, den Fortbestand der Heilung auf dem rechten Obr zu constatiren.

Epikrise. Die eitrige Mittelohrentziundung der Phthisiker pflegt dadurch ausgezeichnet zu sein, dass sich die Krankheit ohne Schmerzen entwickelt, solche auch im weiteren Verlaufe gewöhnlich fehlen, dagegen unter profuser Otorrhoe ein rapider Zerfall des Trommelfells zu Stande kommt. Die ersteren Momente trafen bei dem ersten der oben erwähnten Patienten zu, es hielt sich aber der Ausfluss durchgehends innerhalb sehr mässiger Grenzen und ebensowenig zeigte die Perforation irgend welche Neigung, sich in erheblicherem Maasse zu vergrössern. Im Gegentheil, unter der Jodoformbehandlung schloss sich dieselbe durch eine Narbe, und wenn auch die Entzindung in den Räumen des Mittelohres fortdauerte und das angesammelte Secret sich dem entsprechend stets von Neuem einen Ausgang durch das Trommelfell suchte, so gelang es durch die nämliche Therapie doch immer wieder, die Oeffnung zum Verschluss zu bringen und die Otorrhoe wenigstens vortibergehend $\mathrm{za}$ sistiren. $\mathrm{Ob}$ in dem eitrigen Secret dieses und des anderen Falles Tuberkelbacillen vorhanden waren, vermag ich nicht anzugeben, da beide zu einer Zeit unter meine Beobachtung gelangten, wo hierauf hin noch nicht untersucht wurde. Bei der zweiten Patientin, glaube ich, wüden wir auch wohl ein negatives Resultat erhalten haben; denn aus dem ganzen Krankheitsverlaufe, der Entstehung des Leidens, zu einer Zeit, wo sich wahrscheinlich in den Lungen 
noch gar nichts Abnormes vorfand, und vor Allem aus der leicht und andauernd erzielten Heilung scheint mir der Schluss gestattet zu sein, dass hier zwischen der Affection der Lunge und derjenigen des Ohres gar kein directer Zusammenhang bestand, vielmehr beide unabhängig von einander in einem und demselben Individuum zusammentrafen. Bemerkenswerth ist ferner die Art und Weise, wie die erwähnten Kranken gegen die Borsäure und das Jodoform reagirten. Erstere verschlimmerte entschieden die Entzündung, es stellten sich Schmerzen ein, der Ausfluss nahm an Menge zu, das Trommelfell erschien stärker geröthet und gleich den angrenzenden Theilen der Gehörgangswände an seiner Oberfläche anfgelockert, leicht excoriirt, und einmal erfuhr auch die Perforation eine dentliche Vergrösserung. Eine solche reizende Wirkung der Borsäure ist selten, kommt aber immerhin $a b$ und za vor und zwingt dann zum Aussetzen des Mittels, da eine Gewöhnung an dasselbe unter diesen Umständen nicht stattfindet. Die sonst noch angewandten Medicamente, adstringirende und kaustische Höllensteineinträufelungen, äusserten zwar keinen schädlichen Einfluss, vermochten aber ebensowenig irgend welche Besserung zu Stande zu bringen. Wohl aber trat diese sofort ein, als ich zu den Einblasungen von Jodoformpulver überging. Es sind die erwähnten beiden Fälle bisher die einzigen, in denen ich die Ueberzeugung gewonnen habe, dass das Jodoform mehr geleistet hat als ein anderes der gebräuchlichen Mittel. Im Ganzen erachte ich das Jodoform ftir viel weniger wirksam als die Borsäure und die kaustische Methode, womit allerdings nicht gesagt sein soll, dass man nicht auch durch ersteres in einer Reihe von Otorrhöen Heilung erzielen wird. Es gibt eben zahlreiche Fälle von Otorrhoe, welche der Behandlung überaus leicht zugänglich sind und wo der Ausfluss schon allein bei sorgfältiger Reinigung des Ohres oder dem Gebrauch schwacher adstringirender Lösungen sistirt. Nur ausnahmsweise aber wird das Jodoform noch einen Erfolg erzielen, wo sich die Borsäure and die kaustische Methode unwirksam erwiesen baben, wie bei den oben erwähnten Kranken. Dass es sich hier um mit Phthisis pulmonum behaftete Individuen gehandelt hat, macht die Fälle um so interessanter, da wir ja wissen, dass gerade bei tuberculösen Affectionen das Jodoform sich als besonders heilkräftig erweist. Nur werden wir es leider höchst selten bei Phthisikern mit relativ so gutartigen Formen der Otitis media suppurativa zu thun haben, wie die beschriebenen waren, und 
daher wird auch das Jodoform gleich allen anderen Mitteln in der Regel nicht im Stande sein, etwas zur Heilung dieser Affectionen oder selbst nur zur Erleichterung der vorhandenen Beschwerden beizutragen.

3. Eine seltene Form der acuten eitrigen Mittelohrentzündung.

Während der letzten Jahre habe ich einige Fälle von acutem eitrigem Mittelohrkatarrh beobachtet, welche ein von dem gewöhnlichen Bilde des letzteren vollkommen abweichendes Verhalten, unter sich aber eine grosse Uebereinstimmung in den Symptomen zeigten und auf die ich daher hier etwas näher eingehen will. Es handelte sich im Ganzen um vier Patienten, zwei Männer und zwei Frauen, im Alter von 35 bis 42 Jahren. Die Krankheit hatte sich bei ihnen ohne bekannte Veranlassung und zwar stets einseitig entwickelt, Schmerzen waren weder im Beginn noch späterhin vorhanden und ebenso fehlte jedwede Druckempfindlichkeit. Dagegen maehten sich von Anfang an starke Schwerhörigkeit, Verstopfungsgefühl des Ohres und ein permanentes Rauschen oder Brausen in autfallender Weise bemerkbar. Die Uhr wurde schon jetzt nur noch beim Anlegen oder gar Andriicken an die Ohrmuschel gehört; der Ton der Stimmgabel zog: sich sowohl rom Scheitel und den Zähnen als auch von der Schläfe und dem Warzenfortsatz der gesunden Seite aus nur nach dem kranken Obre hintber. Nach etwa acht Tagen pflegte es zur Perforation des Trommelfells und zum eitrigen Ausfluss zu kommen, jedoch blieb der letztere stets sehr geringfügig, war zu Zeiten kaum bemerkbar, und auch durch Reizmittel, z. B. Höllensteineinträufelungen, gelang es nur ganz vorübergehend, ihn zu einer etwas grösseren Intensität zu bringen. Bei der Untersuchung erwies sich das Trommelfell stark geröthet und geschwollen, sein hinteres Segment vorgewölbt und von gelbröthlicher Färbung, Hammergriff und Processus brevis waren nicht zu erkennen. Die Perforation befand sich ausnahmslos im vorderen unteren Quadranten, sie hatte anfangs nur etwa die Grösse eines Mohnkorns, konnte aber später an Umfang zunehmen, und zwar geschah dieses manchmal förmlich ruckweise, derart dass, nachdem die Oeffnung vielleicht Wochen lang unverändert geblieben, sie sich plötzlich von einem Tage auf den anderen um ibber das Doppelte vergrössert zeigte. Letzteres Verhalten ist iibrigens keineswegs dieser Form der eitrigen Mittelohrentzündung eigenthümlich, vielmehr habe ich die gleiche 
Mittheilungen a. d. Gebiete des einfachen $\mathfrak{u}$. eitrig. Mittelohrkatarrhs. 11

Beobachtung auch in zahlreichen anderen Fällen von Otorrhoe gemacht. Die Luftdouche pflegte keine Veränderung in dem Zustande des Kranken hervorzubringen, sie förderte, trotzdem die Luft in breitem Strome durch die Paukenhöhle hindurchdrang, auch keinen weiteren Eiter heraus. In dieser Art zog sich nun das Leiden, unbeeinflusst durch die Behandlung, Monate lang hin. Dann versiegte die Eiterung allmählich, desgleichen nahm die Röthung und Schwellung des Trommelfells langsam ab und Hammergriff und Processus brevis wurden deutlich, aber damit kam es nicht etwa zur Heilung, vielmehr blieben die Schwerhörigkeit, das überaus lästige Verstopfungsgefühl im $\mathrm{Ohr}$ and die subjectiven Geräusche ungebessert bestehen. In einem der Fälle hatte ich Gelegenheit, den Verlauf bis zum Ende zu beobachten: die Eiterung war sistirt, die linsengrosse Perforation offen geblieben und das Trommelfell präsentirte sich als eine grauweisse, starre Membran, nach innen gezogen, mit deutlichem, sehr schräg gestelltem Hammergriff und stark ausgeprägter hinterer Falte. Was die Therapie anlangt, so habe ich bei diesen Kranken die verschiedensten Mittel versucht, nur leider obne jeden Erfolg. Das Ohr wurde durch Luftdouche, Ausspritzungen, Salzwasserdurchspritzungen durch den Katheter gereinigt, auch ein Versuch mit der trockenen Reinigung desselben wurde gemacht, ferner wandte ich Einblasungen von Borsäure, allein oder mit Tannin gemischt, sowie solche von Jodoform und Calomel an, desgleichen adstringirende Einträufelungen und die kaustische Methode nach Schwartze, sodann wurde örtlich Jod applicirt und innerlich Jodkalium, sowie bei einem Patienten, welcher friiher Syphilis gehabt, Hydrargyrum jodatum flavum verabreicht, aber immer mit dem nämlichen negativen Resultat, der Process schritt unaufhaltsam seinem Ende, der Sklerose, zu. Worauf dieser ungewöhnliche und ungtinstige Verlauf der beschriebenen Form der acuten eitrigen Paukenhöhlenentzïndung zurückzufuhren ist, vermag ich nicht zu sagen. Der eine Kranke hatte allerdings, wie erwähnt, vor vielen Jahren an Lues gelitten, eine zweite Patientin war infolge ihrer Beschäftigung gezwungen, sich fortwährenden Erkältungen auszusetzen, in den beiden anderen Fällen aber lagen derartige Momente nicht vor und tiberhaupt war die Constitution der betreffenden Individuen anscheinend durchweg eine gute. Trotzdem glaube ich, dass wir den Grund des abweichenden Krankheitsverlaufes eher werden in inneren als äusseren Ursachen zu suchen haben, wenngleich ich vorläufig 
nicht in der Lage bin, etwas Bestimmtes zur Stutze dieser Vermuthung anzugeben.

\section{Acuter Mittelohrkatarrh, complicirt mit Erkrankung des Labyrinthes.}

Frau S., 39 Jahre alt, eine nervöse und häufig an Migräneanfällen leidende Dame, kam am 8 . April 1885 in meine Behandlung. Dieselbe hatte sich am 31. März einen oberen Backzahn auf der rechten Seite plombiren lassen. In der Nacht daranf stellten sich heftiges Kopfweh und Erbrechen ein, welche bis zum nächsten Nachmittag andauerten, dann fühlte sich die Patientin wohler, stand auf und hatte uber nichts mehr zu klagen. Am folgenden Morgen bemerkte sie Sausen im rechten Ohre und Druckempfindlichkeit hierselbst, dazu traten etwas später Spontanschmerzen und Schwindel und die Kranke war auf Grund dessen von Neuem genöthigt, sich niederzulegen. Der Schwindel scheint im Ganzen nicht sehr heftig gewesen zu sein, er hielt während mehrerer Tage an, äusserte sich aber noch länger im Momente des Hinlegens. Erbrechen war jetzt nicht vorhanden. Als ich die Patientin sah, hatten die Schmerzen schon wieder etwas nachgelassen, dagegen wurde noch immer iiber starkes permanentes Sausen und Schwerhörigkeit geklagt. Die Uhr wurde nur beim Andrïcken an die rechte Ohrmuschel gehört, vom Warzenfortsatz gar nicht, von der Schläfe links; Stimmgabeln von der rechten Schläfe und Warzengegend rechts, vom Scheitel ausschliesslich rechts. Bei der örtlichen Untersuchung ergab sich folgender Befund. Keine Empfindlichkeit auf Druck. Trommelfell von normaler Stellung und Wölbung; seine Cutissehicht nicht verändert, daher auch der Oberflächenglanz und Lichtkegel normal, wohl aber sah man tiberall, besonders stark im hinteren Segmente, die geröthete Schleimhautschicht hindurchschimmern. Hammergriff deutlich, die denselben begleitenden Gefässe injicirt. Nach Politzer's Verfahren subjectives Gefühl der Erleichterung; Gehör dureh Luft und Knochen unverändert. Die Behandlung bestand in regelmässiger Luftdonche, Jodpinselungen in der Umgebung des Ohres und der innerlichen Verabreichung von Bittersalz.

10. April. Uhr beim Andrücken an die Obrmuschel nicht gehört, vom Knochen unverändert. $\mathrm{Da}$ die Schmerzen noch immer anhielten, sogar wieder an Intensität zugenommen hatten und auch das starke permanente Sausen ein Fortdauern der Ent- 
Mittheilungen a. d. Gebiete des einfachen u. eitrig. Mittelohrkatarrhs. 13

zündung in Paukenhöhle und Labyrinth erkennen liess, wurde innerlich Calomel, zu 0,3 pro dosi, 1 bis 2 mal täglich, verordnet. Von einer localen Blutentziehung nahm jch der Schwäche der Patientin wegen Abstand.

15. April. Es sind im Ganzen funf Calomelpulver verbrancht worden. Keine Schmerzen mehr. Uhr beim Anlegen an die Ohrmuschel, vom Knochen wie oben. Subjective Geräusche und Aussehen des Trommelfells unverändert, nur dass der Oberflächenglanz des letzteren etwas schwächer erschien. Behandlung mit Luftdouche; Jodpinselungen; Bittersalz.

20. April. Noch immer permanentes Sausen. Uhr $4 \mathrm{Cm}$., vom Knochen wie oben. Trommelfellbild beinahe normal, die Röthung fast ganz verschwunden.

27. April. Das Sausen trat jetzt hinter einem Gefühle von Verlegtsein des Ohres zurïck, welches sich aus geringen Anfängen $\mathrm{zu}$ immer grösserer Intensität entwickelt hatte, zur Zeit fast den ganzen Tag über anhielt, sich aber bei horizontaler Lagerung der Patientin regelmässig binnen Kurzem verlor. So fehlte es z. B. in der Nacht vollständig. Im Momente des Niederlegens noch immer leichter Schwindel. Uhr $15 \mathrm{Cm}$., von der Schläfe links, von den Partien zwischen Schläfe und Helix schwach rechts, vom Warzenfortsatz gar nicht gehört. Trommelfellbild normal. - Ordination: Innerlich Jodkalium.

4. Mai. Uhr $20 \mathrm{Cm}$., von der Schläfe schwach rechts, vom Warzenfortsatz 0 .

11. Mai. Verlegtsein des Ohres unverändert. Sausen gering. Uhr $30 \mathrm{Cm}$, von Schläfe und Warzenfortsatz schwach auf der rechten Seite.

16. Mai. Das Verstopfungsgefühl des Ohres fängt an, sich zu verlieren. Kein Sausen. Uhr $40 \mathrm{Cm}$., rechts rom Knochen noch wesentlich schwächer als links.

26. Mai. Das Geftuhl von Verlegtsein stellt sich nur noch selten ein. Kein Sausen. Uhr $60 \mathrm{Cm}$., von Schläfe und Warzenfortsatz so laut wie auf der anderen Seite.

1. Juni. Patientin wird entlassen. Uhr $80 \mathrm{Cm}$. Ohr anbaltend frei. Keine subjectiven Geräusche, kein Schwindel.

Epikrise. In dem vorliegenden Falle handelte es sich um einen acuten Paukenhöhlenkatarrh, complicirt mit Affection des Labyrinthes. Dass neben der Paukenhöhle auch das innere Ohr als mitleidend angenommen werden musste, bedarf wohl keiner näheren Begriundung; dafür sprach die sehr beträchtliche Schwer- 
hörigkeit, das Verhalten der Kopfknochenleitung, die permanenten subjectiven Geräusche, der Schwindel, sämmtlich Erscheinungen, welche sich aus der in ibrer Intensität doch nur mässigen Entziundung des mittleren Obres allein nicht gut erklären liessen und die auch fortdauerten, nachdem jene schon wieder geschwunden war. Interessant waren die Veränderungen, welche die Kopfknochenleitung darbot. Wäbrend die Stimmgabel von Schläfe und Warzenfortsatz aus mit dem kranken Ohr gehört wurde, fehlte die Perception ftur die Uhr von dem rechten Warzenfortsatz ganz und wurde ihr Ticken von der Sehläfe aus nur auf der gesunden Seite vernommen. Dieses dauerte längere Zeit an, sogar als sich die Luftleitung bis zu einem gewissen Grade $(10 \mathrm{Cm}$.) wieder hergestellt hatte. Dann liess sich zuerst eine Stelle zwischen Schläfe und Helix auffinden, von welcher die Uhr schwach auf der gleichen Seite gehört wurde, hierauf folgte die Schläfe und zuletzt der Warzenfortsatz. Die Perceptionsstärke war noch während einer längeren Periode auf der kranken Seite geringer als auf der gesunden. Aufmerksam machen möchte ich sodann auf das Gefuibl von Verlegtsein des Ohres, welches sich nach beinahe vierwöchentlicher Daner der Krankheit einstellte und entsprechend dessen Erscheinen und stärkerer Ausbildung: das Sausen nachliess und sich schliesslich ganz verlor. Diese abnorme Empfindung hielt nur so lange an, als sich die Patientin in aufrechter Stellung befand; sobald sie sich niederlegte, war das Verstopfungsgefuhl regelmässig binnen Kurzem verschwunden und kehrte z. B. auch während der ganzen Nacht nicht wieder. Bei der Anämie des Labyrinthes wird eine solche temporäre Besserung bei und nach horizontaler Lage beobachtet. Indessen möchte es schwer sein, in unserem Falle eine Anämie als Grund der vorhandenen Störungen anzunehmen, und ausserdem bezog sich die Besserung auch nur auf das Verlegtsein, nicht ebenso anf die anderen krankhaften Symptome. Wir kommen hiermit zu der Frage nach der anatomischen Natur der Veränderungen, welche sich in der Paukenhöhle und im Labyrinth ausgebildet hatten, und nach der Ursache, welcher dieselben ihre Entstehung: verdankten. In der Paukenhöhle handelte es sich um einen acuten Katarrh, im inneren Ohr wahrscheinlich um eine Hyperämie, vielleicht auch $\mathrm{um}$ eine leichte Entzündung; beide Alterationen waren wohl einander coordinirt, sie hingen von der nämlichen Ursache $\mathbf{a b}$, die Labyrinthaffection hatte sich nicht erst auf Grund der doch nur mässig starken Mittelohrentziundung 
entwickelt. In ätiologischer Beziehung kennen wir zwei Momente, welche auf die Kranke eingewirkt hatten und die daher möglicherweise mit der späteren Ohraffection in Zusammenhang gebracht werden könnten, d. i, das heftige Erbrechen und das Plombiren des Zahnes. Beide aber lassen sich nicht ohne grosse Schwierigkeiten zur Erklärung herbeiziehen. Die Annahme wäre allerdings gestattet, dass infolge der anhaltenden und starken Brechbewegungen Anomalien der Circulation und vielleicht auch kleine Blutungen in Paukenhöhle und Labyrinth erzengt wurden und dass sich auf Grund dessen dann weitere Störungen entwickelten. Allein unter diesen Umständen wärden wir eine directe zeitliche Verbindung zwischen den Erscheinungen seitens des Ohres und den Brechanfällen erwarten, und doch lag zwischen beiden ein völlig freier Nachmittag und eine Nacht, in denen die Patientin über keinerlei Beschwerden zu klagen hatte. Ziehen wir andrerseits das vorhergegangene Plombiren eines Zahnes auf der Seite des erkrankten Ohres in Betracht, so wissen wir zwar, dass Trigeminusreizungen in der That nicht nur Neuralgien, sondern auch Hyperämie und entzündliche Störungen in der Paukenhöhle zur Folge haben können. Aber es bleibt die Einwirkung auf das Labyrinth unklar, wir mussten denn gerade auf den von Politzer nachgewiesenen Zusammenhang zwischen den Gefässen der Paukenhöhle und des inneren Ohres zurïckgehen, mithin die intralabyrinthären Veränderungen als vom Mittelohr fortgeleitet auffassen, und dazu war doch die Affection des letzteren im Ganzen zu geringfügig. Ferner lag auch zwischen dem Plombiren des Zahnes und dem ersten Auftreten der Ohrsymptome ein freies Intervall von mindestens 40 Stunden. Es bleibt demnach im höchsten Grade zweifelhaft, ob iiberhaupt eines der beiden erwähnten ätiologischen Momente für die Entstehung des Leidens verantwortlich zu machen war and dasselbe nicht vielmehr anf eine andere, uns unbekannte Schädlichkeit als Ursache zurlickgeführt werden musste.

5. Ueber Pulsationen am nicht perforirten Trommelfell.

Pulsationen am nicht perforirten Trommelfell werden allgemein als eine sehr seltene Erscheinung aufgefuhrt. Diese Ansicht besteht zn Recht, soweit sie sich auf die ganz circumscripten Pulsationen bezieht, nicht aber betreffs einer anderen, mehr diffusen Form, welche ich in nicht wenigen Fällen zu beobachten Gelegenheit hatte und die ich daher als ein relativ hänfiges Vor- 
kommniss bezeichnen muss. Es ist bekannt, dass bei intensiven Formen des acuten Mittelohrkatarrhs, wo auch das Trommelfell starken Antheil an der Entzindung nimmt, neben Ekchymosenbildung auf der Oberfläche des letzteren nicht selten auch von hier aus eine blutig-seröse Absonderung stattfindet und als gewöhnlich spärlicher Ausfluss zu Tage tritt. Liegt nun diese Flüssigkeit in dinner Schicht dem Trommelfell an, so kann man, mitunter sogar während längerer Zeit, an derselben ein deutliches Pulsiren wahrnehmen, und zwar entweder nur entsprechend dem vorderen unteren Rande der Membran oder sich auch noch mehr oder minder weit tiber den vorderen unteren Quadranten und selbst die vordere untere Wand des knöchernen Gehörgangs erstreckend. War man aus irgend einem Grunde genöthigt, eine Ausspritzung zu machen, so kann sich bei dem zurtickgebliebenen Spritzwasser die nämliche Erscheinung zeigen. - Von circumscripter Pulsation im Verlaufe eines einfachen Mittelohrkatarrhs habe ich folgenden Fall beobachtet. Ein 66 Jahre alter Musiker war mit starken Schmerzen, Summen und Schwerhörigkeit auf dem rechten Ohre erkrankt und bei der Untersuchung fand sich das Trommelfell von grauröthlicher Färbung und stark geschwollen, der Hammergriff unsichtbar und eine intensivere Röthung in der Umgebung des Processus brevis. Unter Jodpinselungen, Ableitungen auf den Darmkanal und Warmwasserumschlägen liessen die Schmerzen nach, dann aber kehrten sie zwei Tage später in sehr bedeutender Heftigkeit zurück und verbanden sich mit einer nicht unbeträchtlichen Störung des Allgemeinbefindens. Die Localinspection ergab jetzt dicht iber und hinter dem Processus brevis, also im Bereich der Membrana flaccida Shrapnelli, eine stecknadelkopfgrosse, intensiv geröthete und prominente Stelle, deren dünne Bedeckung starke Pulsationen darbot. Es wurde hier ein Einstich gemacht, aber anscheinend nur Blut entleert; bei der Luftdouche war darnach kein Perforationsgeräusch hörbar. Ausserdem wurden zwei Blutegel an den auf Druck empfindlichen Warzenfortsatz applicirt. Der Erfolg dieses Eingriffes war ein sehr glinstiger, die Schmerzen verloren sich sofort, auch die Entziundungserscheinungen nahmen bei regelmässiger Anwendung der Luftdouche allmählich ab und 14 Tage später konnte der Patient als geheilt entlassen werden. Die punktirte Stelle war am nächsten Tage zusammengefallen, sie lag im Niveau ihrer Umgebung und liess keinerlei Pulsationen mehr erkennen. 